\title{
Bibliographie des travaux sur l'oeuvre de Poincaré (2001-2005)
}

\section{Philippe Nabonnand}

\section{Q OpenEdition}

\section{Journals}

\section{Édition électronique}

URL : http://journals.openedition.org/philosophiascientiae/627

DOI : 10.4000/philosophiascientiae.627

ISSN : 1775-4283

\section{Éditeur}

Éditions Kimé

\section{Édition imprimée}

Date de publication : 1 mai 2005

Pagination : 195-206

ISBN : 2-84174-368-3

ISSN : $1281-2463$

\section{Référence électronique}

Philippe Nabonnand, «Bibliographie des travaux sur l'oeuvre de Poincaré (2001-2005) », Philosophia Scientiæ [En ligne], 9-1 | 2005, mis en ligne le 24 juin 2011, consulté le 16 janvier 2021. URL : http:// journals.openedition.org/philosophiascientiae/627 ; DOI : https://doi.org/10.4000/ philosophiascientiae.627 


\section{Extrait du chapitre XXVI de la Philosophie du comme $s i^{1}$}

Hans Vaihinger

Présentation et traduction : Christophe Bouriau Université Nancy 2

Résumé : Nous proposons une traduction française d'un extrait du chapitre XXVI de l'édition populaire de Die Philosophie des Als $\mathrm{Ob}$ de Hans Vaihinger (édition populaire, 1923). L'enjeu de ce texte est de manifester, d'une part, que l'utilisation de fictions est nécessaire au traitement de certains problèmes théoriques et pratiques. D'autre part, il montre qu'utiliser des fictions afin de résoudre certains problèmes théoriques (en l'occurrence, mathématiques) ne conduit pas à l'erreur, pourvu que l'on adopte "la méthode des erreurs antithétiques."

Abstract: We propose a French translation of an extract from chapter XXVI of Vaihinger's Philosophie des Als Ob (popular edition, 1923). The stake of this text is to manifest, on the one hand, that using fictions is necessary to deal with several theoretical and practical problems. On the other hand, it shows that using fictions in order to solve theoretical problems (here, mathematical ones) doesn't lead to mistake, if we resort to "the method of opposite errors."

${ }^{1} \mathrm{Je}$ me base pour cette traduction sur la Volksausgabe (édition populaire) de 1923. La pagination de cette édition est indiquée entre crochets. Il en existe une traduction anglaise, réalisée par C. K. Ogden : The philosophy of 'as if'. A system of the theoretical, practical and religious fictions of mankind, London, Routledge \& Kegan Paul, 1924. Cette traduction contient une précieuse préface de H. Vaihinger, où celui-ci spécifie son pragmatisme par rapport au pragmatisme anglo-saxon. $[N d T]$.

Philosophia Scientice, 9 (1), 2005, 175-194. 


\section{Présentation}

Hans Vaihinger, est né en 1852 près de Tübingen et s'est éteint en 1933 dans la ville de Halle. Auteur d'un volumineux Kommentar zu Kants Kritik der reinen Vernunft (2 vol., 1881-1892), fondateur des Kant-Studien en 1897, de la Kant-Gesellschaft en 1904, Vaihinger prolonge le kantisme dans un sens original. Selon lui, l'idée essentielle de Kant est que certains concepts (Dieu, l'âme, la liberté, etc.) et jugements synthétiques a priori (du type : "l'âme humaine est immortelle", ou : "la nature suit un dessein") se justifient non pas théoriquement, mais comme conditions pour atteindre certaines fins pratiques déterminées. Vaihinger tire Kant vers le pragmatisme, et, sous l'influence de A. F. Lange et de Nietzsche ${ }^{2}$ notamment, radicalise l'idée selon laquelle certaines idées injustifiables théoriquement peuvent néanmoins trouver une justification de type pratique. Son maître ouvrage paru en 1911, $L a$ Philosophie du comme si, remanie et complète une thèse d'habilitation soutenue à Strasbourg en 1877 sous la direction du philosophe positiviste Ernst Laas, portant le titre : Logische Untersuchungen. 1. Teil : Die Lehre von der wissenschaftlichen Fiktion (Recherches logiques. 1ère partie : La théorie de la fiction scientifique).

L'un des principaux intérêts de La Philosophie du comme $s i^{3}$ est de manifester que certaines productions de l'imagination, les fictions, remplissent un rôle positif dans le domaine scientifique également, dans la mesure où elles contribuent à la découverte de la vérité. Si l'on nous autorise un anachronisme, la séparation que fait Bachelard entre la pensée scientifique et la pensée littéraire ou artistique paraîtrait totalement injustifiée aux yeux de Vaihinger, qui unifie les différentes activités humaines à travers leur usage constant du "comme si" : dans quelque domaine que ce soit, la pensée doit sans cesse imaginer, feindre des choses inexistantes pour atteindre ses objectifs ${ }^{4}$ (comme en attestent les exemples mathématiques examinés dans l'extrait proposé).

La démarche de Vaihinger est assez originale car elle va à l'encontre

${ }^{2}$ La philosophie du "comme si" pourrait très bien être lue comme une application rigoureuse de certaines thèses nietzschéennes au champ de l'épistémologie. Rappelons que c'est la lecture de Nietzsche qui a encouragé Vaihinger à publier sa théorie des fictions, qu'il jugeait auparavant trop en avance sur son temps et difficilement acceptable par le grand public.

${ }^{3}$ Le titre complet de l'ouvrage de Vaihinger (1ère éd. 1911) est : Die Philosophie des Als Ob. System der theoretischen, praktischen und religiösen Fiktionen der Menschheit auf Grund eines idealistischen Positivismus. Mit einem Anhang über Kant und Nietzsche. Sur les différentes éditions de l'ouvrage, voir la bibliographie qui suit la traduction.

${ }^{4}$ On retrouvera cette idée chez [Goodman 1994]. 
de l'idée commune, encore répandue chez beaucoup de savants de la fin du XIXe siècle, selon laquelle la découverte de la vérité suppose que soit exclu de la démarche scientifique l'emploi de toute pseudo notion ou pseudo fait ${ }^{5}$.

Vaihinger soutient au contraire que sans le secours de notions sans corrélat réel, ou encore de choses dont on simule l'existence (on se contente de faire comme si elles existaient), toute démarche scientifique serait paralysée. Vaihinger distingue deux sortes de fictions. Celles qu'il nomme "semi-fictions" consistent en un simple écart par rapport au réel donné : par exemple, un classe artificielle provisoirement adoptée par le biologiste, un schéma grossier (négligeant certaines données factuelles) dessiné par un architecte pour résoudre un problème. Celles qu'il nomme "pures fictions", en revanche, ne se contentent pas de contredire le réel tel qu'il est donné, mais contiennent une contradiction interne : par exemple, l'atome comme corpuscule sans dimension est selon Vaihinger une contradiction dans les termes; de même, en mathématiques, la racine des nombres négatifs ou encore l'idée de grandeur infinitésimale donnée ${ }^{6}$.

Vaihinger soutient donc, paradoxalement, que la pensée parvient à résoudre des problèmes théoriques et à maîtriser la réalité grâce à l'emploi de fictions, c'est-à-dire de constructions qui, pourtant, s'écartent de la réalité. Comment cela est-il possible? Comment le détour par le fictif, par le faux, peut-il favoriser la découverte du vrai?

${ }^{5}$ Claude Bernard, par exemple, écrit : "Le raisonnement sera juste quand il s'exercera sur des notions exactes et sur des faits précis; mais il ne pourra conduire qu'à l'erreur toutes les fois que les notions ou les faits sur lesquels il s'appuie seront primitivement entachés d'erreur ou d'inexactitude" [Bernard 1973, 26]. Au contraire, Vaihinger soutient que la "justesse" peut être obtenue en recourant à des notions fausses ou contradictoires, ou encore en faisant comme si existait à titre de "fait exact" ce qui n'existe pas en réalité.

${ }^{6}$ Vaihinger écrit : "Les constructions idéelles sont de pures fictions, au sens strict du terme, lorsqu'elles sont en contradiction non seulement avec la réalité, mais encore avec elles-mêmes (comme par exemple le concept d'atome, de chose en soi). Il faut les distinguer des constructions qui ne contredisent que la réalité donnée, c'est-àdire qui s'en écartent, sans être contradictoires en elles-mêmes (comme, par exemple, une classification artificielle). On peut nommer les dernières demi-fictions ou semifictions. Ces deux types ne sont pas rigoureusement séparés l'un de l'autre, mais reliés par des transitions. La pensée commence d'abord avec des écarts par rapport à la réalité (des demi-fictions), et, devenant de plus en plus audacieuse, elle finit par opérer avec des constructions qui ne contredisent plus seulement le donné, mais qui sont également contradictoires en elles-mêmes" [Vaihinger 1923, 16]. Vaihinger considère la chose en soi kantienne comme une notion contradictoire : en effet, elle est présentée comme l'au-delà du phénomène, auquel la catégorie de causalité ne s'applique pas, et en même temps Kant la définit comme le fondement (Grund) ou la cause (Ursache) du phénomène. 
Le texte qui suit, au moyen de quelques exemples empruntés à différents domaines (droit, mathématiques, logique) montre que la pensée qui utilise des fictions atteint son objectif de deux manières : soit en supprimant purement et simplement du résultat final la fiction qu'elle a introduite pour les besoins du raisonnement (comme dans le cas du juge), soit en neutralisant la première fiction introduite dans le raisonnement par l'introduction d'une seconde fiction qui annule la première (comme dans le cas du mathématicien). Ce second procédé, qui déborde selon Vaihinger la seule sphère des mathématiques, est nommé par lui : "méthode des erreurs antithétiques" 7 . Cette méthode fait comprendre comment la pensée parvient à atteindre la vérité bien qu'elle utilise des fictions.

Il faut préciser que Vaihinger emploie un métalangage philosophique s'exerçant ici sur les langages juridiques, mathématiques et logiques, afin de faire apparaître les similitudes formelles de ces disciplines dans le recours aux fictions et dans la méthode des erreurs antithétiques.

La théorie de la connaissance de Vaihinger est remarquable en ceci qu'elle s'expose à partir d'exemples empruntés à l'histoire des sciences ou aux diverses activités humaines. Ce point me paraît suffisamment exceptionnel pour mériter d'être souligné.

\section{Extrait du chapitre XXVI de La philoso- phie du comme si : "La méthode de cor- rection des écarts arbitraires ou méthode des erreurs antithétiques."}

“(... ) Dans le cas des fictions pratiques, la suppression de ces membres intermédiaires [que sont les fictions] est tout à fait simple : elles disparaissent une fois le but atteint. Certes, elles ne quittent pas la psyché elle-même, mais seulement le processus de la pensée. Par exemple, si le juge utilise la fiction du libre $\operatorname{arbitre}^{8}$, c'est uniquement pour parvenir

\footnotetext{
${ }^{7}$ Vaihinger reprend cette méthode des erreurs antithétiques à Cournot qui, dans [Cournot 1843] mettait à jour la "théorie des erreurs compensées". À propos de cette théorie, Poincaré écrivait dès 1905 qu'elle offrait une réponse aux objections contre les pseudo contradictions de certains raisonnements mathématiques : "La théorie des erreurs compensées de Cournot nous semble répondre de la façon la plus simple aux objections accumulées de tous les philosophes peu versés dans les mathématiques (...)" [Poincaré 1913, 186].

${ }^{8}$ Il s'agit d'une fiction selon Vaihinger au sens d'une supposition fictionnelle, imaginée pour les besoins du raisonnement mais non vérifiable. De la même manière,
} 
à formuler une sanction. Le but est de prononcer une sanction, et la fiction selon laquelle l'homme (en l'espèce le criminel) est libre de ses actes, est ce qui lui permet d'atteindre ce résultat. Il est alors indifférent que l'homme soit libre en réalité. Une fois la sanction prononcée, le concept intermédiaire de liberté s'évanouit, à l'instar du moyen terme dans tout syllogisme. Le syllogisme du juge est le suivant : parce que tout homme est libre de ses actes, tout homme est punissable s'il a enfreint la loi. A est un homme, un homme libre, et il a enfreint la loi. Donc A est punissable. A est d'abord subsumé sous le concept d"homme libre", et, par voie de conséquence, sous celui de "punissable". Mais le concept de liberté s'efface dans la conclusion; il n'a servi qu'à rendre possible le jugement. L'homme est-il réellement libre? Cette prémisse n'est pas examinée par le juge : elle ne vaut que comme une fiction qui lui sert à tirer sa conclusion. S'il n'était pas possible [119] de punir les hommes (les criminels), aucun gouvernement ne serait possible, et c'est en vue de cette fin pratique que la fiction théorique de la liberté a été inventée.

Il en va de même avec la fiction du contrat social : celle-ci ne sert aussi qu'à donner un fondement au droit pénal (et non, comme dans le cas de la liberté, au droit moral de punir). On imagine que chaque habitant d'un pays a fait tacitement un contrat avec la collectivité, celui de respecter les lois, et que la rupture du contrat elle-même est punissable d'après ces lois. Dès lors, si A transgresse ces lois, il rompt le contrat, et, conformément à la loi, mérite une sanction. La fiction théorique du contrat ne sert donc qu'à fonder la pratique du droit pénal. Que ce dernier ne puisse se justifier autrement que par une telle fiction, c'est là un secret de polichinelle de la science politique. En effet, abstraction faite de cette fiction, d'où la collectivité (ou l'un de ses représentants) pourrait-elle tirer le droit de punir les autres hommes? La réponse ne va absolument pas de soi.

Puisqu'on ne saurait réduire la punition à un simple rapport de forces (l'idée que le plus puissant ou la collectivité a le droit de punir les simples particuliers, est dépourvue de tout fondement juridique ou moral), on cherche à fonder théoriquement l'exercice du droit pénal sur la fiction du contrat social. C'est en invoquant cette fiction que, jadis (et, partiellement, aujourd'hui encore), on a cherché à fonder théoriquement le droit du monarque, de la couronne, ou encore, plus récemment, le droit de révolution du peuple. Encore une fois, absolument rien ne permet d'affirmer que l'État détient, de quelque source que ce soit, un droit de

la supposition d'un déterminisme absolu, indispensable au savant pour prévoir les phénomènes, ne saurait prétendre à la moindre validité ontologique. C'est une simple condition de la pratique scientifique. [NdT]. 
punir, que le gouverneur possède un droit de gouverner, et que le peuple a le droit d'engager une révolution. C'est l'idée de contrat qui fournit le moyen terme grâce auquel on peut déduire, théoriquement, les droits que nous venons d'énumérer. Dans la conclusion elle-même, toutefois, le moyen terme [la fiction du contrat] disparaît; une fois achevé le processus de pensée, il s'éclipse.

Avant de poursuivre plus avant cette idée, nous devons nous aviser que la nature des corrections nécessaires [120] varie selon les divers types de fiction, et qu'il y a là une prodigieuse variété de formes. Lorsqu'il s'agit de transpositions illégitimes, par exemple lorsqu'on définit le cercle comme une ellipse, la construction fictionnelle elle-même ne correspond pas à une première erreur, mais à une seconde erreur. En effet, lorsque je dis : "le cercle est une ellipse (...)", c'est une première erreur manifeste, mais lorsque j'ajoute : “(...) dont les foyers ont pour distance 0", la proposition reçoit un sens. De quelle manière? Au gré d'une seconde erreur! En effet, l'idée d'une distance égale à 0 est une erreur, un non sens logique, car une distance égale à 0 n'est précisément pas une "distance". C'est une non occurrence, que l'on considère ici simplement comme une occurrence accompagnée de sa négation. On a donc deux "erreurs" : la première est l'affirmation que "le cercle est une ellipse", et elle est compensée par la seconde erreur consistant à poser "une distance égale à 0", qui, littéralement, présente la même contradiction que la définition du cercle comme ellipse. Ici aussi, l'idée d'une distance égale à 0 constitue le moyen terme qui disparaît une fois atteint le résultat. Le syllogisme est le suivant : "Toute figure dont les deux foyers sont à une certaine distance l'un de l'autre, est une ellipse. Le cercle possède deux foyers dont la distance est égale à 0 . Donc le cercle est une ellipse". On constate que le moyen terme fictionnel disparaît du résultat final. Or c'est précisément ce résultat, cette généralisation du concept d'ellipse, cette subsomption du cercle sous une figure qui lui est tout à fait contraire, que l'on voulait obtenir. Cette démarche révèle également la subjectivité de tous nos termes classificatoires. En l'adoptant, je peux en définitive subsumer les uns sous les autres des concepts qui entretiennent un rapport de contrariété, ce qui ne va pas sans contredire la logique ordinaire.

Toute une série de corrections consiste dans le fait de récuser, une fois le calcul terminé, la modification que l'on a fait initialement subir à la situation réelle. Ce procédé intervient particulièrement en mathématiques. Prenons par exemple le problème suivant :

Comment diviser la ligne a en deux parties : $x$ et $a-x$, de sorte que $x(a-x)$ présente la plus grande quantité possible?

Ce problème historique parut longtemps insoluble, [121] jusqu'à ce 
que Fermat le résolve par l'artifice suivant : il remplace $x$ par $x+e$, c'est-à-dire par une partie entièrement arbitraire, plus grande que celle recherchée [plus grande que $x$ ]. La formule :

$$
x^{2}(a-x)
$$

devient ainsi :

$$
(x+e)^{2}(a-x-e)
$$

Fermat pose une équivalence entre les deux formules en faisant comme si elles étaient égales, bien qu'elles ne le soient pas. En effet leurs valeurs respectives sont manifestement différentes : par exemple, $6^{2}(9-6)$ donne 108 , tandis que $(6+1)^{2}(9-6-1)$ donne 98 . Fermat nomme cette équivalence adaequalitas (Diophanti $\pi \alpha \rho \iota \sigma \mathrm{o} \tau \eta \zeta$ ), et il pose les équations suivantes :

(I) $x^{2}(a-x)=x^{2} a-x^{3}$

(II) $(x+e)^{2}(a-x-e)=\left(x^{2}+2 e x+e^{2}\right)(a-x-e)=a x^{2}+2 a e x+$ $a e^{2}-x^{3}-2 e x^{2}-e^{2} x-e x^{2}-2 e^{2} x-e^{3}$

Il pose ensuite, comme on l'a vu, que (I) $=(\mathrm{II})$. On obtient donc :

$$
\begin{aligned}
& x^{2} a-x^{3}=a x^{2}+2 a e x+a e^{2}-x^{3} \\
&-2 e x^{2}-e^{2} x-e x^{2}-2 e^{2} x-e^{3} \\
& \\
& 2 a e x+a e=3 e x^{2}+3 e^{2} x+e^{2} \\
& 2 a x+a e=3 x^{2}+3 x e+e^{2}
\end{aligned}
$$

Mais comment continuer?

Fermat annule la première erreur en déclarant que $\mathrm{x}+$ e n'était qu'une simple fiction pour amorcer le calcul. Il faut bien en effet que (I) soit égal à (II); or ce n'est possible que si l'on $\operatorname{pose}^{9}: e=0$. Toutes les

\footnotetext{
${ }^{9}$ En admettant que la différence entre $x$ et $x+e$ correspond à une grandeur très petite, l'équation est presque correcte. En admettant que la différence est aussi minime que possible, l'équation devient plus correcte encore. Mais si l'on admet que $e=$ un infiniment petit, la différence devient infiniment petite; admet-on pour finir que $e=0$, la différence, elle aussi, égale 0 . La grandeur $e$, bien qu'elle ne soit rien, est donc imaginée comme un quelque chose; quelque chose est introduit et admis comme une réalité. C'est là également un préliminaire du calcul différentiel.
} 
expressions incluant e sont alors annulées, ce qui donne [122] :

$$
\begin{aligned}
2 a x & =3 x^{2} \\
2 a & =3 x \\
\frac{2}{3} a & =x
\end{aligned}
$$

Admettons par exemple que la longueur de la ligne $a=12$. En appliquant la formule citée, on obtient alors : $x=8, a-x=4$. Du reste, c'est à cette seule condition que $x^{2}(a-x)$ présentera la quantité maximale recherchée, à savoir $8^{2}(4)=256$. Toutes les autres manières de diviser la ligne donnent un résultat inférieur : par exemple, $7^{2}(5)=245$; $6^{2}(6)=216 ; 5^{2}(7)=175$; etc.

Cet exemple remarquable offre une image typique de toute pensée fictionnelle. et discursive. L'astuce de Fermat est la suivante : la grandeur imaginaire $x+e$ diffère de la grandeur $x$ si $e$ est un nombre réel, mais elle n'en diffère pas si $e$ est égal à 0 . Toute la méthode repose sur un quaternio terminorum, au sens où $e$ est d'abord envisagé comme une grandeur réelle (s'ajoutant à $x$ ), avant d'être réduit à 0 .

Comme la mise en équation de $x^{2}(a-x)$ avec $(x+e)^{2}(a-x-e)$ n'est, en toute rigueur, pas possible, Fermat parle dans ce premier moment d'une adaequalitas, c'est-à-dire d'une égalité approximative, non parfaite. Il n'en procède pas moins comme si l'égalité était parfaite, quand bien même le code rigoureux des mathématiques et de la logique refuse que $x$ puisse jamais égaler $x+e$. Par cette procédure, c'est-à-dire en interposant la fiction $x+e$, censée être égale à $x$, il va tout de même obtenir un résultat correct!

Comment Fermat s'y est-il pris au juste? Au cours de sa procédure, il a annulé la première erreur en supprimant purement et simplement la quantité auxiliaire $e$. L'égalité qu'on obtient dans l'équation terminale n'est plus une égalité imaginaire, comme l'était la première, mais une égalité réelle. L'invention de la fiction $(x+e)$ et l'emploi de la méthode des opérations antithétiques [la seconde opération annulant la première] permettent ainsi d'atteindre un résultat extrêmement important.

La pensée mathématique adopte exactement la même méthode, même si elle se présente sous une forme plus simple, pour résoudre certaines équations du second degré. Prenons l'équation : $x^{2}+p x=q$.

Tant que l'équation se présente sous cette forme, la pensée ne peut rien entreprendre. Elle ne pourra avancer que grâce à la méthode des opérations antithétiques. Comment [123] la pensée s'y prend-t-elle ici? 
Elle introduit la quantité auxiliaire $\left(\frac{p}{2}\right)^{2}$, et commence par poser :

$$
x^{2}+p x+\left(\frac{p}{2}\right)^{2}=q
$$

Toutefois cette formulation est une erreur. Aussi une opération antithétique est-elle immédiatement effectuée : le même symbole est ajouté de l'autre côté de l'égalité. On a donc :

$$
x^{2}+p x+\left(\frac{p}{2}\right)^{2}=q+\left(\frac{p}{2}\right)^{2}
$$

À présent l'équation peut être résolue, car :

$$
\begin{aligned}
\left(x+\frac{p}{2}\right)^{2} & =q+\left(\frac{p}{2}\right)^{2} \\
x+\frac{p}{2} & =\sqrt{q+\left(\frac{p}{2}\right)^{2}} \\
x & =\sqrt{q+\left(\frac{p}{2}\right)^{2}}-\frac{p}{2}
\end{aligned}
$$

Comment la pensée mathématique a-t-elle obtenu ce résultat? En introduisant la quantité auxiliaire $\left(\frac{p}{2}\right)^{2}$ et en l'ajoutant des deux côtés de l'équation. En ajoutant $\left(\frac{p}{2}\right)^{2}$ également du côté droit, j'annule l'erreur commise du côté gauche. C'est une règle mathématique générale : ne pouvant modifier quantitativement un côté de l'équation sans commettre une erreur, sans détruire l'équation, je neutralise aussitôt cette erreur par une erreur antithétique.

Formellement, ce procédé est presque identique à celui de Fermat. Dans la terminologie mathématique, on nomme cela un artifice ou une astuce [Kniff]. Le second procédé cité présente une image claire de la semi-fiction : les données du problème sont altérées, mais la nature spécifique de l'objet traité exige [124] ici un correctif immédiat, un rétablissement qui, dans le cas des autres semi-fictions, n'intervient que plus tard.

Dans le premier exemple cité, le correctif ou le rétablissement n'intervenait qu'après coup. Cet exemple offrait l'image d'une pure fiction, au sens où l'on commettait une contradiction. En effet, de deux choses l'une : ou bien $x^{2}(a-x)=(x+e)^{2}(a-x-e)$, donc $x+e=x$ et $e=0$ (l'artifice consiste à calculer avec 0 , car 0 n'est pas un nombre); ou bien $x$ n'est pas égal à $x+e$, mais alors l'équation est fausse. En un sens, elle est fausse également dans le premier cas, dans la mesure où $x+0$ n'a aucune signification : de fait cette expression ne dit rien de plus que 
$x$, et que pourrait bien signifier $0^{2}$ ? De toute manière, nous ne pouvons absolument pas échapper à la contradiction. Mais la subtilité des mathématiques consiste précisément à calculer avec des symboles dénués de signification (tels que le 0 ) en faisant comme si il s'agissait de véritables nombres.

J'affirme que toutes les opérations fictionnelles, d'un point de vue formel, sont parfaitement identiques aux opérations mathématiques citées. Il est évident qu'elles adoptent toutes le même premier moment formel, qui consiste en une simple altération de la réalité (sans que cela implique encore contradiction). Dans le second exemple cité, le membre de l'équation $x^{2}+p x$ est altéré par l'ajout arbitraire $\left(\frac{p}{2}\right)^{2}$ (naturellement cet ajout ne doit pas être complètement arbitraire, mais entretenir une certaine relation avec le membre en question). De la même manière la psyché, dans les méthodes fictionnelles que nous avons précédemment citées (classification artificielle, fiction abstractive ou "néglective", etc.), altère ce qui est donné. Et pour que le résultat final soit juste, il faut ici aussi procéder à une opération antithétique. Dans notre exemple mathématique cette opération corrective a lieu aussitôt, dans les autres cas, elle intervient plus tardivement.

Je ne prétends pas de cette manière réduire les fonctions logiques aux fonctions mathématiques, mais montrer l'identité formelle des méthodes scientifiques à l'œuvre dans les différents domaines de la science, en particulier de celles qui atteignent leur but par une altération des données, par un écart arbitraire par rapport à la réalité. L'exemple mathématique que j'ai choisi est simplement [125] le plus transparent. Il fait bien voir comment la pensée peut progresser précisément en s'écartant de la réalité. La fonction logique se trouve confrontée aux données brutes, au matériel de la pensée; sans se laisser rebuter par elles, elle modifie arbitrairement le donné, met les idées en mouvement et redresse très tranquillement à la fin l'erreur qu'elle a commise au début. Nous voyons ici dans tout son éclat ce que les divers artifices logiques ont d'identique au plan formel.

On a complètement oublié aujourd'hui que Berkeley s'est attaché à ramener le calcul différentiel au schéma de calcul de Fermat, en montrant (sans citer l'exemple de Fermat) que de cette manière on parvient à expliquer le calcul différentiel. Sa conclusion est que procède en commettant une double erreur. Les objections de Berkeley se trouvent dans son ouvrage oublié The analyst, et sont développées en détail en cinquante sections [Berkeley 1734]. Ce n'est pas le moindre des mérites de Berkeley que d'avoir pointé les contradictions contenues dans la méthode des fluxions. Seulement, il a commis lui-même une erreur très étrange. 
Il montre parfaitement par quel artifice le mathématicien atteint son résultat, à savoir, en commettant une double erreur. Mais au lieu de voir que cette trouvaille absolument géniale - beaucoup plus profonde que les développements de Leibniz et de Newton sur le sujet - permet d'obtenir un résultat correct et justifie l'application d'une telle méthode, il rejette entièrement cette dernière comme illogique, en tant qu'elle contrevient au code traditionnel de la logique. L'histoire lui a donné tort, car aujourd'hui encore, recourant aux mêmes artifices, on commet délibérément les mêmes erreurs, les mêmes contradictions, et de manière parfaitement justifiée. Mais Berkeley a réitéré son erreur sous une forme plus étrange encore. Après avoir montré de manière très pertinente, et avec une clairvoyance géniale, que presque tous les concepts fondamentaux des mathématiques étaient contradictoires, il conclut que les mathématiciens n'ont aucun droit de dénoncer les éléments incompréhensibles et mystérieux du christianisme, puisque leur propre discipline souffre précisément de la présence d'éléments de cette nature. Le calcul des fluxions illustre manifestement à ses yeux l'un de ces éléments incompréhensibles, puisqu'il le rejette comme contradictoire. Cependant le bon évêque de Cloyne, en authentique britannique, ne prend pas conscience [126] qu'il lui faut alors rejeter du même coup tous les concepts fondamentaux de la théologie chrétienne!

Il avait pourtant la solution du problème à portée de main. Nous assistons au spectacle étrange, qui ne se répétera sans doute pas de si tôt dans l'histoire de la science, d'un penseur qui tient à sa disposition la solution d'un problème, mais qui ne voit pas le problème lui-même!

Voici comment les choses se sont passées. Berkeley est irrité parce que quelques mathématiciens "free-thinkers" se sont exprimés en termes sceptiques au sujet des éléments incompréhensibles de la dogmatique chrétienne. Il dépense alors tous ses efforts à montrer que les mathématiciens doivent d'abord balayer devant leur propre porte : "Celui qui habite une maison de verre ne devrait pas jeter de pierres autour de lui." Son unique aspiration est de montrer que le calcul des fluxions souffre lui-même de contradictions. En suivant cette voie, c'est tout à fait par hasard qu'il découvre et établit la méthode mathématique dont nous parlons, qui consiste à redresser une première erreur commise au moyen d'une seconde erreur, de sorte que l'on obtient un résultat juste!

Berkeley était mu par une aspiration non scientifique. Le vrai problème, à l'époque comme aujourd'hui, est le suivant : comment se faitil qu'en mathématiques, on puisse atteindre un résultat juste avec des contradictions du type de celles qui sont impliquées dans le calcul des fluxions? Au lieu de cerner ce problème, Berkeley ne cherche qu'à dé- 
noncer les contradictions en question! Mais son entreprise le conduit audelà de ce qu'il voulait, puisqu'il en découvrant la méthode des erreurs antithétiques, il trouve la réponse à une question qu'il ne s'est nullement posée. Cette "réponse" aurait cependant dû le conduire formuler la vraie question. Un pareil cas est assurément rarissime dans l'histoire des sciences.

Comme on l'a vu, la solution du problème [comment peut-on atteindre un résultat juste en employant des fictions?] est la suivante : un résultat correct peut être obtenu grâce à la "méthode des erreurs antithétiques."

Il faudrait consacrer une étude spéciale au détail de cette découverte extrêmement intéressante de Berkeley sur le calcul des fluxions (découverte que Drobisch et Carnot ont faite à leur tour après lui). De mon point de vue, cette méthode de calcul découle tout naturellement de notre principe, et ne présente qu'une fraction de la méthode fictionnelle en général. On introduit des quantités auxiliaires [127] qui sont supprimées par la suite, ce qui n'est possible, en mathématiques, que par des opérations antithétiques. La véritable clef de l'énigme consiste à considérer $d x$ et $d y$ tantôt comme équivalents à 0 , tantôt comme des quantités réelles - quand bien même très petits. Telle est la solution mathématique du problème. Quant à savoir pourquoi l'on obtient le résultat juste avec la fiction de $d x$, de $d y$ et de $d s$, on peut répondre de manière entièrement différente, selon que l'on souhaite traiter la question de manière purement mathématique, ou bien l'expliquer au moyen de concepts discursifs. En langage mathématique, on peut dire que cette fiction est un simple artifice, et que les éléments $d x$, etc., disparaissent à la fin parce qu'une erreur opposée, la quaternio, les annule. Assimilés initialement à quelque chose de réel, ces termes sont finalement réduits à 0 . Logiquement et discursivement parlant, on peut dire que nous avons recours au concept d"infiniment petit" qui est une simple construction conceptuelle discursive, construction qui, dans le calcul mathématique lui-même, est considérée comme un simple symbole. Dans le calcul différentiel, traité de manière purement mathématique, cet élément n'est nullement nécessaire. Du reste, on a tenté à plusieurs reprises d'évacuer l'infiniment petit du calcul mathématique. En fait, point n'est besoin de l'éloigner du calcul, puisque, à bien y réfléchir, il ne s'y trouve pas vraiment, comme Berkeley l'a pertinemment montré.

Toute l'affaire repose sur un artifice mathématique. L'interprétation discursive de la procédure, en revanche, cherche à la justifier en montrant à juste titre que le concept d'infini en question est illusoire. Le calcul de Fermat peut lui aussi se justifier de cette manière : on peut dire que $x+e$ 
ne diffère qu'infiniment peu de $x$, raison pour laquelle ils peuvent être mis en équation. Aux yeux du mathématicien, cette justification n'est pas valable : aussi petit que soit $e, x+e$ reste différent de $x$.

L'approche mathématique, si elle est pure, sans éléments discursifs, peut considérer que la méthode de Fermat et la méthode infinitésimale sont exemptes du concept d'infinité. De même que e est un simple symbole pour 0 , pris d'abord pour égal à quelque chose de réel avant d'être ramené à 0 , de même $d s, d x, d y$ sont d'abord pris pour quelque chose de réel et de très petit (non pas infiniment petit) avant d'être ramenés à 0. C'est par ce mécanisme simple et ingénieux que l'on obtient [128] le résultat. À cette fondation ou justification de type mathématique, s'oppose la justification de type discursif qui, elle, emploie le concept abusif d'infiniment petit.

Il est parfaitement exact que les méthodes en question peuvent se dispenser de l'infinitésimale, mais pour autant elles ne sont pas exemptes de contradiction : en effet, elles calculent avec 0 comme avec un nombre, ou encore, elles commencent à poser $e$ et $d s$ comme très petits, puis comme égaux à 0 . Ce sont des artifices qui doivent l'obtention d'un résultat juste à la méthode des erreurs antithétiques. On commence par introduire $e$, puis on l'exclut, et il en va de même pour la différentielle.

L'explication au moyen du concept de l'infiniment petit relève exclusivement de l'approche discursive, et il y a des chances que partout ailleurs l'on parvienne également à se passer de ce concept, reconnu ici comme superflu. Mais même si l'on n'y parvenait pas, il demeurerait à titre de fiction. L'important est de montrer que c'est au moyen de fictions que l'on obtient le bon résultat, par la méthode des erreurs antithétiques. En effet, même lorsque nous considérons $e$ et $d s, d x, d y$ comme des infiniment petits, la méthode est exactement semblable à la précédente : nous introduisons à nouveau une construction fictionnelle, au sens où l' "infiniment petit" est un non-sens logique, un mixte de "rien" et de "quelque chose". Et tout le secret de l'affaire réside dans cette méthode ridiculement simple consistant à prendre ce concept tantôt pour quelque chose, tantôt pour rien.

L'infiniment petit est une fiction. Il est vrai qu'au moyen de cette fiction (justifiée par la méthode des erreurs antithétiques), le donné est pour ainsi dire dissous, mais c'est la seule façon de faire progresser le calcul. C'est en effectuant des opérations antithétiques que la pensée progresse, et la synthèse de ces opérations antithétiques en un même concept engendre la fiction, qui n'est que le symptôme de ces opérations ou erreurs antithétiques. Ainsi les contradictions contenues dans ces concepts sontelles à la fois élucidées et justifiées. Ce qui importe le plus, ce ne sont pas 
ces concepts eux-mêmes, mais les opérations antithétiques qui trouvent en eux leur expression, et par lesquelles la pensée progresse. [129] Nous avons pu voir sur des exemples mathématiques spécifiques comment un tel progrès était possible, et nous aurons encore l'occasion de nous y arrêter dans la suite. Tout progrès de la pensée repose exclusivement sur ces opérations et erreurs antithétiques; toute progression logique consiste entièrement dans ce mouvement de va-et-vient, qui n'est pas une ligne droite mais un louvoiement constant contre les vents défavorables.

J'ai tenté ci-dessus de montrer, sur des exemples mathématiques spécifiques, avec quelle ingéniosité l'âme procédait pour résoudre certains problèmes difficiles : elle contourne simplement la difficulté et cherche à atteindre son but indirectement. Les exemples considérés étaient frappants, et les seuls à pouvoir nous dévoiler le véritable mécanisme de la pensée, son mécanisme psychique. S'il est vrai que toutes les catégories et tous les concepts généraux, comme on l'a vu, ne sont à leur tour que des fictions, est-ce à dire qu'on assiste avec eux à un processus similaire? Oui, naturellement, comme je l'ai indiqué et comme je veux à nouveau le montrer. Nous avons vu que par l'interposition d'une catégorie, on ne produisait pas seulement l'illusion d'une compréhension, mais l'on introduisait un certain ordre dans les phénomènes, rendant possible le traitement des data de l'expérience. Du reste, je ne vois pas comment la division des phénomènes en sections catégorielles pourrait être autre chose qu'une division artificielle. D'un point de vue pratique, cette division est tout à fait valable. Toutefois, qui peut prétendre aujourd'hui qu'elle génère une quelconque connaissance, qui peut nier qu'elle conduit à des incongruités et à des contradictions, à ce que Lotze nomme des "termes impossibles"?

Notre table des catégories correspond à une classification purement artificielle des choses, et le principe de cette division est exclusivement l'analogie que nous établissons entre certaines relations constantes de succession et de coexistence dans les phénomènes et certaines relations subjectives. Certaines relations dominantes sont retenues pour former des centres de similitude autour desquels sont regroupés les autres cas similaires. [130] Toutefois, au cours du raisonnement, ces centres de similitude sont de fait éliminés.

L'attitude à l'égard de la seconde grande fiction de la fonction logique, celle des concepts généraux, n'est pas très différente : ils sont eux aussi supprimés à l'issue du raisonnement, comme le furent ci-dessus " $e$ " et $d s, d x, d y$. En effet, ces constructions fictionnelles que nous interposons dans le raisonnement sont dépourvues de signification effective, objective. Suivons le cours d'un raisonnement pour analyser le méca- 
nisme psychologique qui est en jeu. Prenons le vieil exemple scolaire qui conclut que Socrate est mortel parce que chaque homme, parce que l'homme en général, est mortel. La tâche consiste ici à attribuer à un cas particulier ce qui a pu être observé sur des milliers de cas similaires. Nous avons donc :

M-P L'homme est mortel

$\underline{\mathrm{S}-\mathrm{M}}$ Socrate est un homme

S-P Socrate est mortel.

Qu'avons-nous fait au juste? À l'aide du moyen-terme "homme", nous avons effectué une inférence dont le résultat est : Socrate est mortel. Le moyen-terme lui-même a disparu du résultat. Ce qui importe en effet, c'est seulement le cas individuel Socrate : c'est de comparer ce phénomène particulier avec une série innombrable d'autres cas, afin d'être en mesure d'inférer ou de percevoir un rapport de coexistence et de succession commun à ce cas (Socrate) et aux autres cas, lorsque le cas particulier Socrate se présente à notre perception. En réalité, cette conclusion est une hypothèse formée par analogie : parce que beaucoup d'hommes, c'est-à-dire tous les cas connus, sont mortels, Socrate est également mortel. Mais c'est le moyen-terme "homme" qui soutient et facilite cette analogie -sur laquelle repose en fait tout notre savoir.

Une fois le résultat atteint, le moyen-terme disparaît. Ici, les opérations antithétiques consistent, premièrement, dans le fait de former le concept général d"homme", [131] deuxièmement, dans le fait de subsumer sous lui Socrate lui-même : c'est de cette manière qu'il devient possible de laisser tomber, au final, le moyen terme "homme".

Nous avons donné aux opérations antithétiques le nom d'erreurs antithétiques. Nous souhaitons maintenant en faire une application particulière. Par cette application, il se pourrait que nous trouvions un résultat inattendu et tout à fait différent. La première erreur consiste dans la formation du concept de "l'homme en général". Que signifie cet "en général" ? C'est une pure imagination, une fiction, une construction idéelle fictionnelle. À partir de quantités innombrables d'hommes offerts à l'observation, émerge peu à peu une image générale, un type, un schéma, dans lequel sont rassemblées les "caractéristiques" les plus générales de ces phénomènes similaires. Cette image n'est qu'une construction idéelle : il n'existe en réalité que des hommes particuliers. Nous nommons cette construction concept général d'"homme". Puisqu'une telle construction ne renvoie à rien d'existant, en toute rigueur la proposition : "l'homme est mortel" est fausse, car seuls sont mortels les hommes particuliers, les individus $A, B, C, D,(\ldots)$ Une déclaration sur "l'homme" en général est logiquement fausse, c'est un écart par rapport à la réalité donnée, qui ne 
nous donne jamais "l'homme" comme tel.

Mais la mineure : S est M est également fausse, car elle met sur le même plan un nouvel être individuel et un concept général. M comme concept général (dans la majeure) diffère de Mabc (dans la mineure), qui, lui, contient en outre Socrate ( $a b c$ désigne, dans la terminologie de Lotze, la série des cas individuels contenus dans le concept). Nommons ce second M : M1. La mineure dit : S est M. Or ce n'est pas vrai, car S est M1.

De même que nous avions plus haut un quaternio terminorum dans le cas du e (dans le calcul de Fermat, e était d'abord identifié à quelque chose de réel, puis réduit à 0 ), il en est de même ici : nous avons aussi un quaternio, et c'est lui qui abrite la méthode des opérations antithétiques. Premièrement, tous les individus observés sont rassemblés au gré d'une abstraction arbitraire dans un M général, qui comprend les propriétés observées sur ces individus passés. Puis, à l'inverse, M1 [incluant Socrate] est abusivement identifié à ce $\mathrm{M}$, et une erreur antithétique est ainsi commise. [132] Mais cette seconde erreur permet de compenser l'écart arbitraire opéré dans la majeure, et d'abandonner le moyen-terme "homme" dans la conclusion.

Par conséquent toute inférence de ce type, dont le moyen-terme est un concept général, repose sur un quaternio terminorum.

Dans l'usage des concepts généraux également, la méthode et le mécanisme de la pensée se ramènent à la méthode générale des opérations antithétiques que nous venons d'observer. Nous devons ajouter un corollaire à ce qui vient d'être dit : les expressions qui n'ont pas encore été compensées par l'opération antithétique sont, en toute rigueur, aussi fausses que l'était l'équation $x^{2}(a-x)=(x+e)^{2}(a-x-e)$, avant d'être redressée par l'opération antithétique $[e=0]$.

Ceci s'applique à toutes les propositions générales : la seule fonction d'une proposition générale est de servir d'intermédiaire au cours d'une opération : la proposition "L'homme est mortel", comme l'ont déjà avancé de nombreux sceptiques, est fausse parce que "l'homme" n'a pas d'existence réelle. C'est un terme sans réalité, requérant son complément, comme une demi-page en quête de son autre moitié.

À juste titre, Lotze (comme Steinthal avant lui) a fait la même observation à propos de tous les concepts contenus dans les catégories. Steinthal attire plusieurs fois l'attention sur le fait que le jugement fournit avant tout une résolution de la tension introduite dans la pensée par la formation de concepts individuels. Nous accordons naturellement, d'après notre théorie, que tous les concepts, toutes les relations mises en 
catégories sont des fictions. La tension et la contradiction vient de ce que l'on pose isolément un terme singulier de la relation, par exemple "arbre", "vert". Séparés, isolés, ces termes sont de simples fictions, tandis que si je dis : "cet arbre est vert", la tension est résolue à travers le jugement.

Le véritable principe qui confère un caractère achevé aux concepts isolés et aux jugements généraux, c'est l'idée qu'ils ne sont que des moyens en vue d'une fin, sans laquelle ils sont sans valeur : considérer ces moyens en faisant abstraction de leur fin ne conduit qu'à une tension et à une contradiction.

[133] Toutefois ce n'est là qu'un cas particulier d'un phénomène plus général, impliqué dans toutes les fictions. Nous avons souligné à plusieurs reprises que pris et traités séparément, ces membres intermédiaires que sont les fictions n'étaient que des coquilles sans noyau. Dès qu'une fiction est envisagée indépendamment du sol où elle a grandi et de la fin qu'elle sert, c'est une coquille sans noyau, comme $\sqrt{-1}$, ou encore $d y, d x$, e, etc., qui sont des termes contradictoires, des concepts illusoires, qui n'ont de vie qu'en relation à ce qui est effectif : sans cette relation, ils sont morts. Abstraction faite de leur finalité, ils sont sans valeur. Des périodes entières se sont contentées de semblables coquilles, comme le Moyen-âge, qui s'est consacré à des concepts coupés de tout lien à la réalité vivante, concepts qui pourtant ne remplissent leur fonction qu'à travers ce lien. S'attacher aux fictions pour elles-mêmes est toujours sans valeur et néfaste, car elles ne reçoivent de valeur que rapportée à une fin. Cette règle générale, qui découle avec une absolue nécessité de l'essence de la fiction elle-même et qui, en même temps, permet d'expliquer beaucoup de choses dans l'histoire de la science, régit les observations faites cidessus, qui n'en sont qu'une application particulière. Les concepts et les jugements généraux construits grâce à eux n'ont naturellement aucune valeur, à moins d'être rapportés aux phénomènes particuliers et à leur contexte. Le concept requiert le jugement parce qu'en lui-même il est une simple construction idéelle incomplète. Le jugement individuel corrige une faute, à savoir la décomposition du phénomène au moyen des catégories, puisqu'il opère une réunification. Dans notre exemple précédent : "l'arbre est vert", ou "le sucre est blanc", nous voyons que pris en eux-mêmes, les termes "sucre" et "blanc" sont de simples fictions. En revanche le jugement : "le sucre est blanc" exprime un fait réel. Certes nous avons vu qu'un tel jugement général, si on le considère d'un point de vue supérieur, [134] était faux à strictement parler. En effet, seuls existent des morceaux particuliers de sucre, et non le sucre en général.

Mon analyse ne prend sa véritable importance et sa pleine signification que lorsqu'on l'applique aux grandes idées, celles que les philosophes 
affectionnent particulièrement : Dieu, la liberté, l'immortalité, la chose en soi, l'absolu, etc., mais également à toute un série d'idées et de méthodes qui, pour la première fois, sont considérées ici à partir d'un point de vue d'ensemble. Ces idées et ces méthodes prennent leur véritable valeur à l'instant même où toute valeur de vérité doit leur être refusée. Le véritable intérêt de notre recherche se manifeste dans l'application rigoureuse de cette théorie à certains concepts courants, à certaines idées fameuses, mais aussi au monde des idées tout entier; c'est de cette manière seulement que le positivisme critique atteint un développement conséquent et complet. Sont fictionnels, à mes yeux, non seulement les divers concepts, non seulement toute une série de méthodes, non seulement la pensée discursive, mais encore l'univers des idées dans son ensemble. Ce qui est réel et ce qui le reste, c'est la constance que nous pouvons observer dans les phénomènes et leurs relations; tout le reste n'est qu'une illusion avec laquelle joue la psyché.

\section{Références}

Berkeley, G.

1734 The analyst. A discourse addressed to an infidel mathematician, 1734.

BERNARD, C.

1865 Introduction à l'étude de la médecine expérimentale, 1865; Paul Foulquié, éditions de l'école (rééd.), Paris, 1973.

Carnot, L.

1803 Géométrie de la position, Duprat, Paris, 1803.

Cournot, A.

1843 Exposition de la théorie des chances et des probabilités, Hachette, 1843 ; rééd. Vrin, Paris, 1984, avec notes critiques de Bernard Bru.

Drobisch, M. W.

1834 Grundzüge der Lehre von höheren numerischen Gleichungen, Leipzig, 1834 (8e éd.).

Goodman, N.

1994 Reconceptions en philosophie, tr. fr. J.-P. Cometti et R. Pouivet, PUF, Paris, 1994.

LANGE, A. F.

1866 Histoire du matérialisme et critique de son importance à notre époque, 1866 ; tr. fr. B. Pommerol, 2 t., C. Reinwald et Cie, 1879, 
Paris.

LEIBNIZ, G. W.

1684 Nova methodus pro minimis et maximis, Acta Eruditorum, Leipzig, 1684.

1989 Naissance du calcul différentiel, 26 articles des Acta Eruditorum, Introduction, traduction et notes de Marc Parmentier. Paris, Vrin, 1989.

LOTZER, R. H.

1874-1879 System der Philosophie : I. Drei Bücher der Logik (Leipzig, 1874); II. Drei Bücher der Metaphysik (Leipzig, 1879).

Newton, I.

1740 La méthode des fluxions et des suites infinies, tr. fr. M. de Buffon, librairie Debure l'aîné, Paris, 1740 (consultable sur Gallica, BNF); fac simile, A. Blanchard, Paris, 1994.

OGDen, C. K.

1924 trad. anglaise de l'ouvrage de Vaihinger : The philosophy of 'as if'. A system of the theoretical, practical and religious fictions of mankind, London, Routledge \& Kegan Paul, 1924.

PoINCARÉ, H.

1913 Dernières pensées, Flammarion, Paris, 1913.

Steinthal, H.

1861 Charakteristik der Hauptsächlichsten Typen des Sprachbaues, F.Dümmler, Berlin,1861.

VAIHINGER, H.

1881-1892 Kommentar zu Kants Kritik der reinen Vernunft, 2 vol., 1881-1892 ; 2e éd. assurée par Raymund Schmidt, Stuttgart, 1922 ; réimpression de la 2e éd. : Scientia Verlag Aalen, 1970.

1911 Die Philosophie des Als Ob. System der theoretischen, praktischen und religiösen Fiktionen der Menschheit auf Grund eines idealistischen Positivismus. Mit einem Anhang überKant und Nietzsche, Leipzig, Felix Meiner, 1911 (1ère éd.), 1913 (2e), 1918 (3e), 1920 (4, 5 et $6 \mathrm{e}), 1922$ (7 et $8 \mathrm{e}), 1927$ (9 et 10e); réimpression de la 9/10e éd. : Scientia Verlag Aalen, 1986.

1923 Die Philosophie des Als Ob. System der theoretischen, praktischen und religiösen Fiktionen der Menschheit auf Grund eines idealistischen Positivismus. Mit einem Anhang überKant und Nietzsche, 
Leipzig, Felix Meiner, 1923 (Volksausgabe : édition populaire), 1924 (2e éd.). 